paramount importance. I am confident that giving students a framework of standard questions and phrases and then allowing them the combined privacy and space to practice the use of such in a safe learning environment will improve their confidence in sexual history taking.

\section{P189 "... GIVING SOMETHING BACK TO THE GAY COMMUNITY BY TAKING PART": GAY AND BISEXUAL MEN'S UNDERSTANDINGS OF PARTICIPATION IN BEHAVIOURAL RESEARCH}

Nicola Boydell*, Gillian Fergie, Shona Hilton, Lisa McDaid. MRC/CSO Social and Public Health Sciences Unit, University of Glasgow, Glasgow, Scotland, UK

\subsection{6/sextrans-2015-052126.233}

Background/introduction Studies exploring public participation in health research have not, to date, included the perspectives of gay and bisexual men taking part in behavioural surveillance research. Understanding factors which motivate men to participate in behavioural research, and their perceptions of feedback on anonymous HIV antibody tests are important in the design of future studies.

Aim(s)/objectives The aim of this qualitative study was to gain insight into men's motivations for participation in the Gay Men's Sexual Health Survey (GMSHS), and their understandings of, and views on, HIV testing as part of the survey.

Methods Semi-structured telephone interviews were conducted with 29 gay and bisexual men who participated in the 2011 GMSHS. Men were recruited in 13 licensed premises on the commercial 'gay scene' in Edinburgh and Glasgow. Data were analysed thematically, focusing on motives for participation and perceptions of not receiving individual feedback on HIV status.

Results Most men expressed sophisticated understandings of the purpose of behavioural research and distinguished between this and individual diagnostic testing for HIV. Men's accounts suggested a shared understanding of participation in research as a means of contributing to 'community' HIV prevention efforts. Among the men interviewed feedback on HIV status was not deemed crucial.

Discussion/conclusion Continuing to engage with gay and bisexual men, and practitioners working within these communities, is vital to engendering trust in, and support for, future behavioural research. This is particularly important during the process of developing new and innovative research strategies. Further research is needed to explore men's perceptions of participation in research, and their perspectives on receiving feedback on testing, within wider contexts.

\section{P190 WE DON'T NEED NO SEX EDUCATION: DO YOUNG PEOPLE VALUE THE KNOWLEDGE THEY GAIN FROM SCHOOL AND SEXUAL HEALTH SERVICES?}

Jonathan Shaw*, John Sweeney. Blackpool Sexual Health Services, Blackpool, UK

\subsection{6/sextrans-2015-052126.234}

Introduction There remains ongoing debate regarding the value of sex education in schools and if today's young people subsequently rely on alternative resources to learn about sex and relationships.
Aims As a provider of sexual health services for young people aged under 25 we wanted to establish if there was an expectation amongst service users for us to provide sex education.

Methods Questionnaires were distributed to all service users between April and September 2014. Questions were designed to assess how sexual knowledge had been acquired, and which method of knowledge acquisition was most valued.

Results 179 service users completed questionnaires. 160 were female, 149 were heterosexual. Median age was 18.6 years.

177 (98.9\%) reported receiving sex education at school which predominantly covered reproduction and contraception. Comparing methods of knowledge acquisition advice from friends was the most valued $(84,46.9 \%)$, followed by sexual partners $(57,31.8 \%)$ and family $(56,31.3 \%)$. Formal sex education was only valued by 34 (19.0\%), with sexual health clinic advice valued by 32 (17.9\%).

The desire for more sex education at school was mixed with $74(41.3 \%)$ wanting more and 106 (59.2\%) requesting no change or were unsure. $46(25.7 \%)$ requested an increase in education from our clinic.

Conclusion Service users valued knowledge gained from peers and family over current methods of formal sex education with no significant desire to increase current educational provision. Sexual health services should engage young people in discussions regarding this peer-based learning to reinforce good sexual health and dispel inevitable myths.

\section{P191 SURVEY OF GENITAL DERMATOLOGY TRAINING AMONGST GENITOURINARY MEDICINE (GUM) SPECIALIST REGISTRARS}

${ }^{1}$ Anna Hartley, ${ }^{2}$ Christine Bates*, ${ }^{3}$ Parameswaran Sashidharan. ${ }^{1}$ Barts Health NHS Trust, London, UK; ${ }^{2}$ Royal Liverpool University Hospital, Liverpool, UK; ${ }^{3}$ Homerton University Hospital, London, UK

\subsection{6/sextrans-2015-052126.235}

Introduction There has been no recent review of genital dermatology (GD) training for GUM trainees. The 2010 GUM specialist registrar curriculum states specific learning objectives that trainees should meet by CCT.

Aim In order to evaluate and improve training, the BASHH GD Special Interest Group (SIG) conducted an online survey to assess specialist registrar training in GD.

Methods The survey was designed through Survey Monkey and cascaded to trainees across the UK in 2014.

Results 42 trainees responded, representing several deaneries (50\% London) and grades. $68 \%$ of trainees receive GD training through adhoc clinical teaching; $85 \%$ through formal lectures. $26 \%, 32 \%, 37 \%$ have attended specialist GD clincs by gynaecologist, GUM physician, dermatologist respectively. Mean confidence in managing specific conditions varied from 5 (vulval pain syndromes) to 7.5 (fungal infections) (1-10 confidence scale). $47 \%$ were $\geq 7 / 10$ confident in topical steriod use (1-10 confidence scale). Independently able to perform procedures: $21 \%$ punch biopsies, $63 \%$ fungal scrapings, $15 \%$ curettage.

$50 \%$ of trainees are satisfied with GD training with $69 \%$ feeling they will be adequately trained by CCT. 58\% would like a formal qualification in GD to be available.

Discussion Training in GD is variable with mixed confidence in diagnosis, treatment and practical procedures. Many trainees feel 
training could be improved with requests for a formalised attachment, formal qualification and greater training in practical procedures.

The BASHH GD SIG, in liaison with BASHH, aims to optimise GD training for registrars. Plans for improved resources are in progress, including a practical skills course and e-learning.

\section{P192 SUDDENLY YOU'RE ON YOUR OWN, AND YOU'RE OUT THERE IN THE BIG WORLD: MIDDLE-AGED ADULTS' SEXUAL RISK-TAKING BEHAVIOURS WITHIN THE CONTEXT OF LIFE-COURSE TRANSITIONS}

${ }^{1,2}$ Jenny Dalrymple* ${ }^{1}$ Joanne Booth, ${ }^{1}$ Paul Flowers, ${ }^{1}$ Karen Lorimer. ${ }^{1}$ Glasgow Caledonian University, Glasgow, UK; ${ }^{2}$ NHS Greater Glasgow and Clyde, Glasgow, UK

\subsection{6/sextrans-2015-052126.236}

Background/introduction While sexual activity, including partner change, is known to continue throughout the life course, there is a paucity of qualitative evidence on how adults over 45 years engage with risk for sexually transmitted infections (STIs), limiting the scope for effective health promotion among this age group.

Aims/objectives The research aimed to explore older adults' sexual risk-taking behaviour within the context of sexuality in later life.

Methods A qualitative in-depth study involving 31 interviews with middle aged heterosexual men and women aged 45 to 65 , recruited from sexual health clinic and community settings.

Results Vulnerability to STI risk emerged around key life course transitions, including following divorce, separation and bereavement. Some spoke enthusiastically of embracing sexual freedom and pleasure within a perceived changed culture, resulting in frequent partner change; however, many found themselves 're-engaging' with their sexual careers within an unfamiliar gendered landscape. Lacking an (ageing) body confidence led to the prioritisation of intimacy over STI risk; condoms were viewed as being for birth control and therefore mostly unnecessary, or linked with casual sex and lack of trust. STIs were commonly considered to be a young person's concern.

Discussion/conclusions Information provision alone will not be enough to counter the complexities of navigating the dramatically different sexual landscape these older adults find themselves within compared to their youth, particularly those who have emerged from long-term relationships. A separately focussed approach to STI prevention taking account of life course experience, ageing and cultural change is advocated.

\section{P193 DEVELOPMENT OF A HANDHELD POINT OF CARE MOLECULAR DIAGNOSTIC DEVICE FOR SEXUALLY TRANSMITTED INFECTIONS}

${ }^{1}$ Ruth Mackay*, 1,2Pascal Craw, ${ }^{1}$ Manoharanehru Branavan, ${ }^{3}$ Tariq Sadiq, ${ }^{1}$ Wamadeva Balachandran. 'Brunel University London, Uxbridge, Middlesex, UK; ${ }^{2}$ Environmental Genomics, Oceans and Atmosphere Flagship, Commonwealth Scientific and Industrial Research Organisation, Hobart, Tasmania, Australia; ${ }^{3}$ St. George's, University of London, London, UK

\subsection{6/sextrans-2015-052126.237}

Background/introduction Brunel DoCLab is part of the eSTI2 Consortium which is developing electronic self-testing and portable instruments for sexually transmitted infections using nucleic acid amplification test technologies. We have designed a point of care test platform that integrates a proprietary sample collection device directly with a microfluidic cartridge. A low cost benchtop real-time isothermal amplification platform has been developed capable of running six amplifications simultaneously.

Aim(s)/objectives To evaluate the sample preparation and isothermal amplification within the low cost diagnostic platform. Methods The microfluidic device incorporates passive mixing of the lysis-binding buffers and sample. Cell lysis, within the cartridge, is conducted using a chemical method and nucleic acid purification is done on an activated cellulose membrane. Isothermal amplification was conducted using recombinase polymerase amplification (RPA).

Results Preliminary results have shown extraction efficiencies for this new membrane of $69 \%$ and $57 \%$ compared to the commercial Qiagen extraction method of $85 \%$ and $59.4 \%$ for 0.1 $\mathrm{ng} / \mu \mathrm{L}$ and $100 \mathrm{ng} / \mu \mathrm{L}$ salmon sperm DNA respectively spiked in phosphate buffered solution. Extraction experiments in the passive mixer cartridges with lysis and nucleic acid purification showed extraction efficiency around $80 \%$ of the commercial Qiagen kit. The platform is capable of detecting Chlamydia trachomatis genomic DNA within 10 min using RPA for 100,000 copies $/ \mu \mathrm{L}$.

Discussion/conclusion The work presented here shows a low cost, rapid nucleic acid extraction, isothermal amplification and detection platform for diagnosing C. trachomatis. Work is ongoing to fully integrate the sample-in to result platform for rapid diagnosis of STIs using genital samples.

\section{P194 COST-EFFECTIVENESS OF CHLAMYDIA TESTING IN SCOTLAND}

${ }^{1}$ Lesley Wallace*, ${ }^{2}$ Katharine Looker, ${ }^{2}$ Katy Turner. ${ }^{1}$ Health Protection Scotland, Glasgow, UK; ${ }^{2}$ University of Bristol, Bristol, UK

\subsection{6/sextrans-2015-052126.238}

Background/introduction Scottish chlamydia testing guidelines target symptomatic and high-risk asymptomatic individuals. Recent publications, indicating a low risk of progression to serious chlamydia-related outcomes, particularly tubal factor infertility (TFI), question the validity of high levels of opportunistic testing especially among asymptomatic individuals.

Aim(s)/objectives To examine cost-effectiveness of current chlamydia testing to prevent TFI among those aged 15-24 in Scotland using cost per Quality-Adjusted Life Years (QALYs) gained and to consider alternative testing strategies.

Methods A compartmental deterministic model of chlamydia infection in those aged 15-24 in Scotland was developed to examine the impact of testing coverage and partner notification (PN) on number and cost of TFI cases prevented. Cost-effectiveness calculations were informed by best estimates of the QALYs lost due to TFI.

Results At $16.8 \%$ baseline testing coverage (laboratory data), $4.4 \%$ prevalence (NATSAL-3) and assumed PN rate of 0.4, the total testing cost is $£ 5.4$ million. This is estimated to prevent 258 TFI cases each year in young women. The cost per QALY gained is $£ 40,034$ compared with no testing, using a mid-range health state utility value (HSUV) for TFI $(0.76( \pm 0.24))$ and PID $(0.9( \pm 0.22))$. A $50 \%$ reduction in current testing would result in higher chlamydia prevalence and 84 more TFI cases.

Discussion/conclusion Current chlamydia testing activities in Scotland do not appear cost-effective. However, the model is sensitive to several parameters, particularly the HSUV and there are uncertainties in the current testing costs and progression to 